\title{
OBSTRUCTIVE SLEEP APNOEA (OSA)
}

Sleep disordered breathing is a common entity; of which obstructive sleep apnoea (OSA) is one of the important one to address. Patient usually presents with day time somnolence, inability to concentrate, snoring, unrefreshing sleep, apnoea or hypopnoea during sleep; with or without arousal. Usually patient is a loud snorer but he is not aware of the symptoms; it is the bed partner who takes the person to a physician.

Some time physicians suspect patients to have OSA; if anybody having uncontrolled blood pressure inspite of getting adequate treatment, uncontrolled diabetes inspite of having adequate insulin or other hypoglycemic agents, resistant heart failure having adequate treatment, nocturia which is otherwise unexplained; even impotence when other common causes have been excluded.

Pathophysiology of OSA is as follows: during sleep muscle tone decreases, so dilator muscles of the pharynx loses its tone $\&$ tongue fall back posteriorly; completely occluding the air passage, leading to airway obstruction. Oxygen can't go to the lungs, leading to hypoxia, causing vigorous contraction of respiratory $\&$ abdominal muscles, which leads to arousal of patient $\&$ reliving obstruction. This cycle occurs repeatedly during sleep in a patient with OSA.

Physical examination may reveal the following findings; high body mass index (BMI), Increased neck circumference, microganthia, Malampathi score $^{1}$ may be low or high, tonsiler enlargement, long uvula, nasal turbinate hypertrophy.

Gold standard for the diagnosis of OSA is overnight polysomnography (PSG) in a sleep laboratory. It usually includes, simultaneous recording of Electro encephalogram (EEG), Electro myogram (EMG), Electro occulogram (EOG), Nasal airflow, Chest movement, abdominal movement, Oxygen saturation ( SPo2), Electrocardiogram (ECG), leg movements $\&$ snoring.

By PSG we see the apnoea (complete cessation of breathing, more than 10 seconds), hypopnoea (complete cessation of breathing, less than 10 seconds), oxygen desaturation during the events, arousals, periodic leg movements, We can diagnose the severity of OSA by apnoea hypopnoea index (AHI- number of apnoeas \& hypopnoeas per hour). AHI $<5$ is normal, $5-15$ is mild OSA, $15-30$ is moderate OSA and $>30$ is severe OSA. Central sleep apnoea can be differentiated from OSA by PSG. If OSA is confirmed, an overnight CPAP (Continuous positive airway pressure) titration is done by an auto CPAP to estimate the pressure requirement to overcome the obstruction.

Home sleep study is also recommended now a days; for suspected OSA patients. It looks only four parameters (Nasal airflow, Chest movement, abdominal movement, SPo2). If home sleep study is normal but patient is symptomatic; an overnight PSG is recommended.

Moderate to severe OSA requires treatment with CPAP during sleep at night; at least 4 hours/ day. If pressure requirement is more than 15 $\mathrm{cm}$ of water, a BiPAP (bi level positive airway pressure) is recommended. A proper fitting face or nasal musk is required to reduce leaks. A humidifier is also required in dry environment as it may cause dryness in mouth \& nose. Nasal

Correspondence : Prof. Salma Rouf, Professor of Obstetrics and Gynaecology, Dhaka Medical College

Received: 10 February $2019 \quad$ Revision: 02 March 2019

Accepted: 30 Mach 2019

DOI: https://doi.org/10.3329/jdmc.v28i1.45747 
decongestants may be prescribed for blocked nose. Mouth breathers should be prescribed with full face musk.

Though CPAP therapy is the gold standard for the treatment of OSA but adherence is only 35$45 \%$. Other therapy includes dental appliances \& surgery (Mandibular advancement surgery, Tonsillectomy, Turbinectomy, Uvuolo palato pharyngoplasty). Recently hypoglossal nerve stimulation therapy is also used for the treatment of $\mathrm{OSA}^{2}$.

OSA is very common in western world, untreated OSA may lead to increased chances of motor vehicle accidents. In Bangladesh exact scenario is not known, but our younger generation is getting obese; in future there may be an epidemic of OSA!

J Dhaka Med Coll. 2019; 28(1) : 1-2

\section{Professor (Dr.) Md. Mohiuddin Ahmad}

Professor \& Head

Department of Respiratory Medicine

Dhaka Medical College \& Hospital

e-mail: dr.mohiuddin.ahmad@gmail.com

\section{References:}

1. Mallampati SR. Clinical sign to predict difficult tracheal intubation. Can Anesth Soc J. 1983 May; 30 (3 pt 1):316-7.

2. Barbe F, Masa JF. Hypoglossal nerve stimulation for obstructive sleep apnoea. Eur respire J. 2013 Feb;41(2):257-8. 